\title{
Fully exploiting the power of 2D NMR J-resolved spectroscopy
}

Qing Zeng, Jinyong Chen, Chaoqun Zhan, Yanqin Lin, ${ }^{*}$ and Zhong Chen

Fujian Provincial Key Laboratory of Plasma and Magnetic Resonance, State Key Laboratory for Physical Chemistry of Solid Surfaces, Department of Electronic Science, Xiamen University, Xiamen 361005, China

*Corresponding author. Email: linyq@xmu.edu.cn

\section{Table of Contents}

Figure S1. Experimental results for quinine Figure S2. Experimental results for estradiol

Figure S3. Comparison of CASCADE and real-time SERF experiments

Figure S4. Pulse sequences for CASCADE with real-time J-upscaling acquisition

Figure S5. CASCADE spectrum with J-upscaling for butyl methacrylate

Figure S6. Results of inosine for demonstration of suppression of strong coupling artifacts

Pages S8 - S14. Codes of pulse sequence 


\section{Experimental results for quinine}

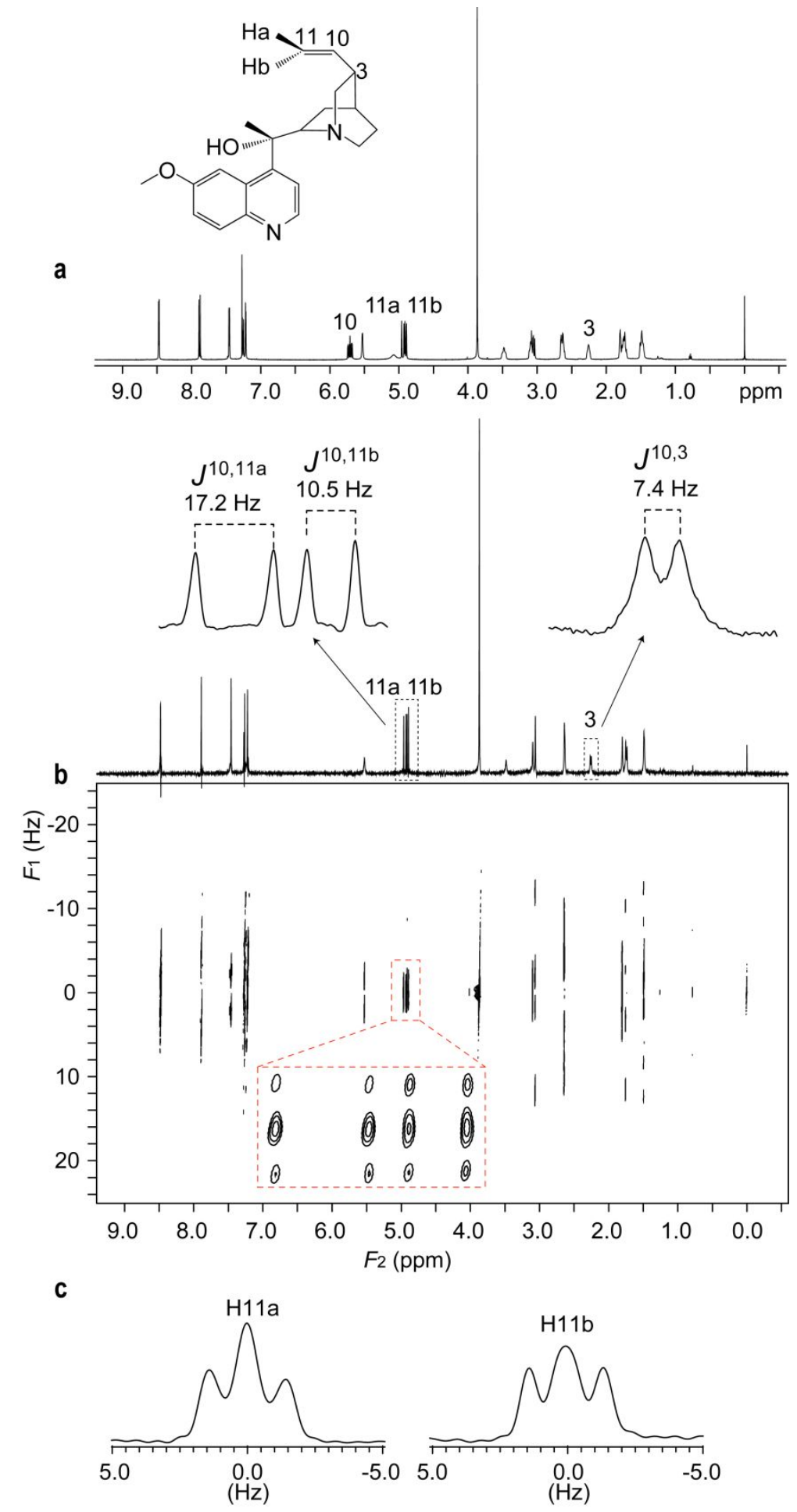

Figure S1. (a) Normal 1D ${ }^{1} \mathrm{H}$ NMR spectrum and molecular structure of quinine (200 mM in DMSO- $d_{6}$ ). (b) Absorption-mode CASCADE spectrum, with $F_{2}$ projection on the top and close-up views of signals indicated by dotted boxes. (c) $F_{1}$ projections of protons $\mathrm{H} 11 \mathrm{a}$ and $\mathrm{H} 11 \mathrm{~b}$. Selective $180^{\circ}$ pulses were exerted on proton $\mathrm{H} 10$, and coupling constants $J^{10,11 a}, J^{10,11 b}, J^{10,3}$ can be measured. The inset in $b$ shows close-up views of signals of protons $\mathrm{H} 11 \mathrm{a}$ and $\mathrm{H} 11 \mathrm{~b}$. 


\section{Experimental results for estradiol}
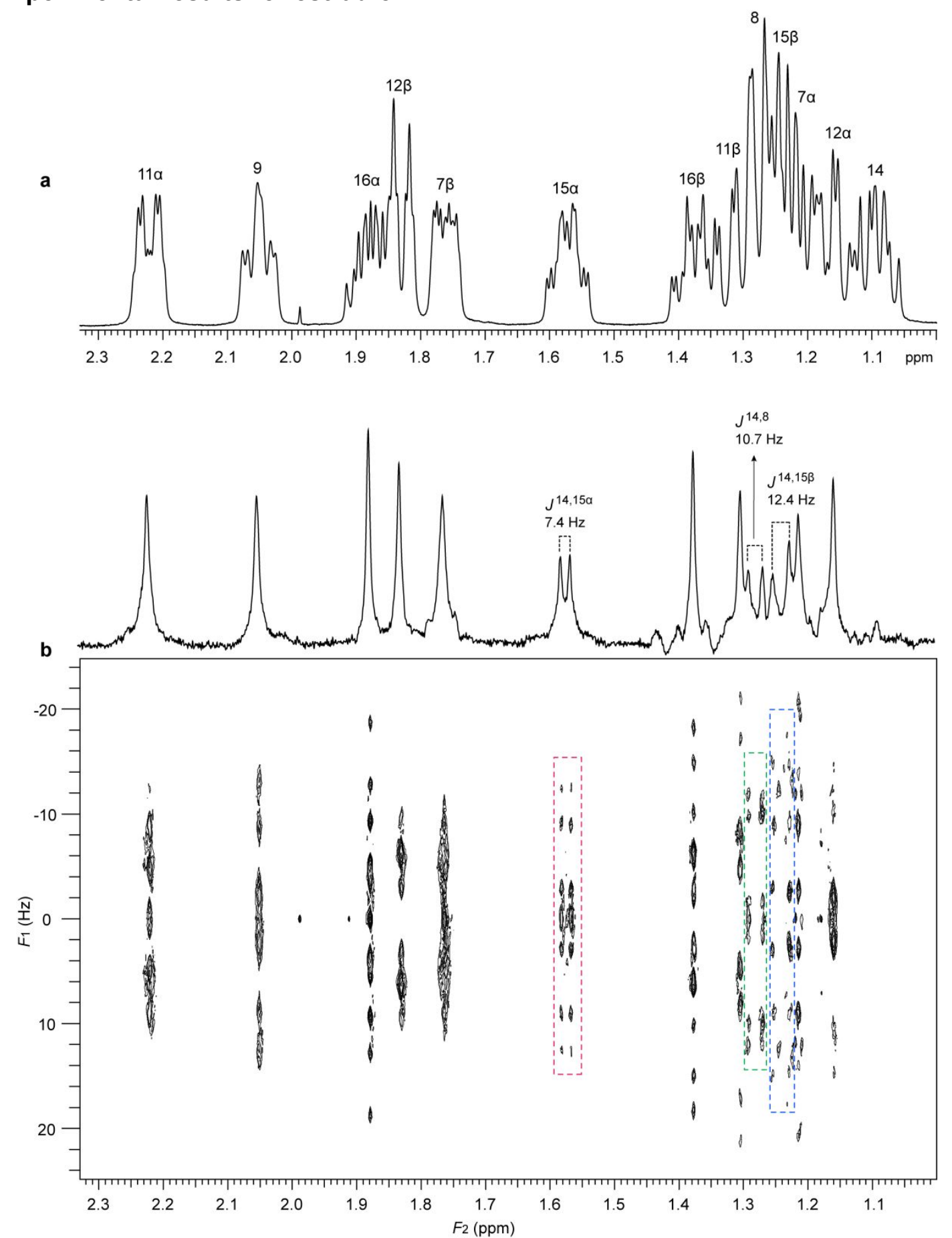

Figure S2. (a) Normal 1D ${ }^{1} \mathrm{H}$ NMR spectrum of estradiol (100 mM in DMSO- $d_{6}$ ). (b) Absorption-mode CASCADE spectrum, with $F_{2}$ projection on the top. Selective $180^{\circ}$ pulses were exerted on proton $\mathrm{H} 14$, and coupling constants $\mathrm{J}^{14,15 \alpha}, \mathrm{J}^{14,8}, J^{14,15 \beta}$ can be measured. Signals of protons $\mathrm{H} 15 \alpha, \mathrm{H} 8$ and $\mathrm{H} 15 \beta$ are also indicated by dotted boxes in the 2D spectrum. 


\section{Comparison of CASCADE and real-time SERF experiments}

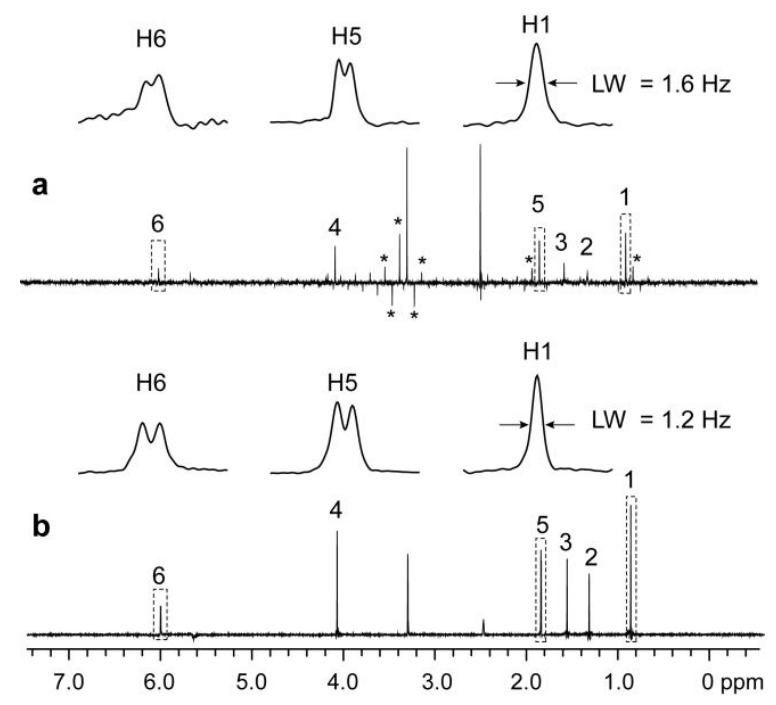

Figure S3. Experimental results for butyl methacrylate. Proton $\mathrm{H} 7$ was selected for investigation by selective $180^{\circ}$ pulses. (a) Real-time SERF spectrum. (b) The $F_{1}$ projection of CASCADE spectrum from Fig. 2b. Artifacts and broader lines can be seen in the real-time SERF spectrum. Linewidths (LWs) are also shown. Several intense artifacts are indicated by asterisks. 
Pulse sequences for CASCADE with real-time $J$-upscaling acquisition
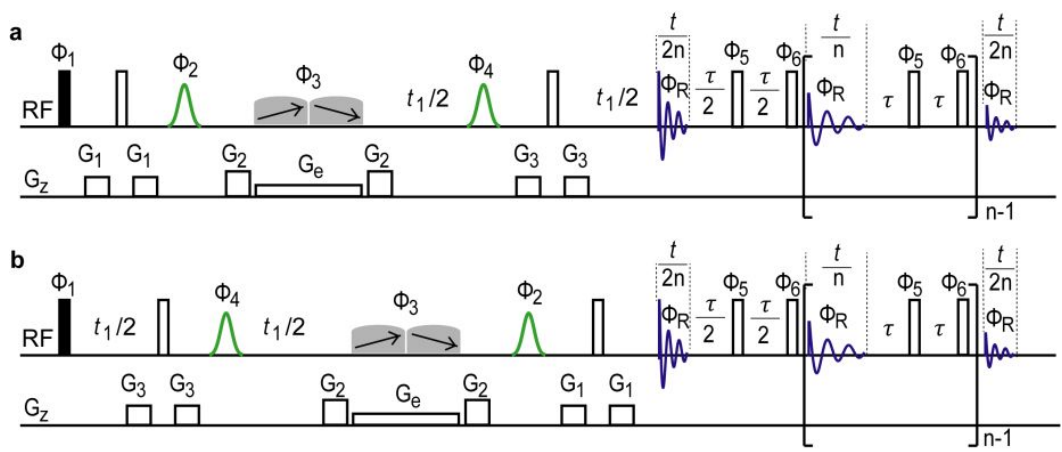

Figure S4. Pulse sequences for CASCADE with real-time J-upscaling acquisition. Sequences in $a$ and $b$ are used for normal and revered $J$ spectra, respectively. Filled rectangle shapes on channel RF represent hard $90^{\circ}$ pulse. Unfilled rectangle shapes on the channel RF are hard $180^{\circ}$ pulses. Gaussian shapes on the channel RF are selective $180^{\circ}$ pulses. Shaped pulses with diagonal arrows are low-angle frequency-swept chirp pulses which sweep frequencies in opposite directions. Rectangle-shaped z-direction gradients are shown on the $G_{z}$ channel. The phase cycle is: $\Phi_{1}=y,-y,-y, y, \Phi_{2}=x, \Phi_{3}=$ $\mathrm{x}, \mathrm{x},-\mathrm{x},-\mathrm{x}, \Phi_{4}=\mathrm{x}, \mathrm{y},-\mathrm{x},-\mathrm{y}, \Phi_{5}=\mathrm{x},-\mathrm{x}, \Phi_{6}=-\mathrm{x}, \mathrm{x}, \Phi_{\mathrm{R}}=-\mathrm{y}, \mathrm{y}, \mathrm{y},-\mathrm{y}$. 


\section{CASCADE spectrum with J-upscaling for butyl methacrylate}
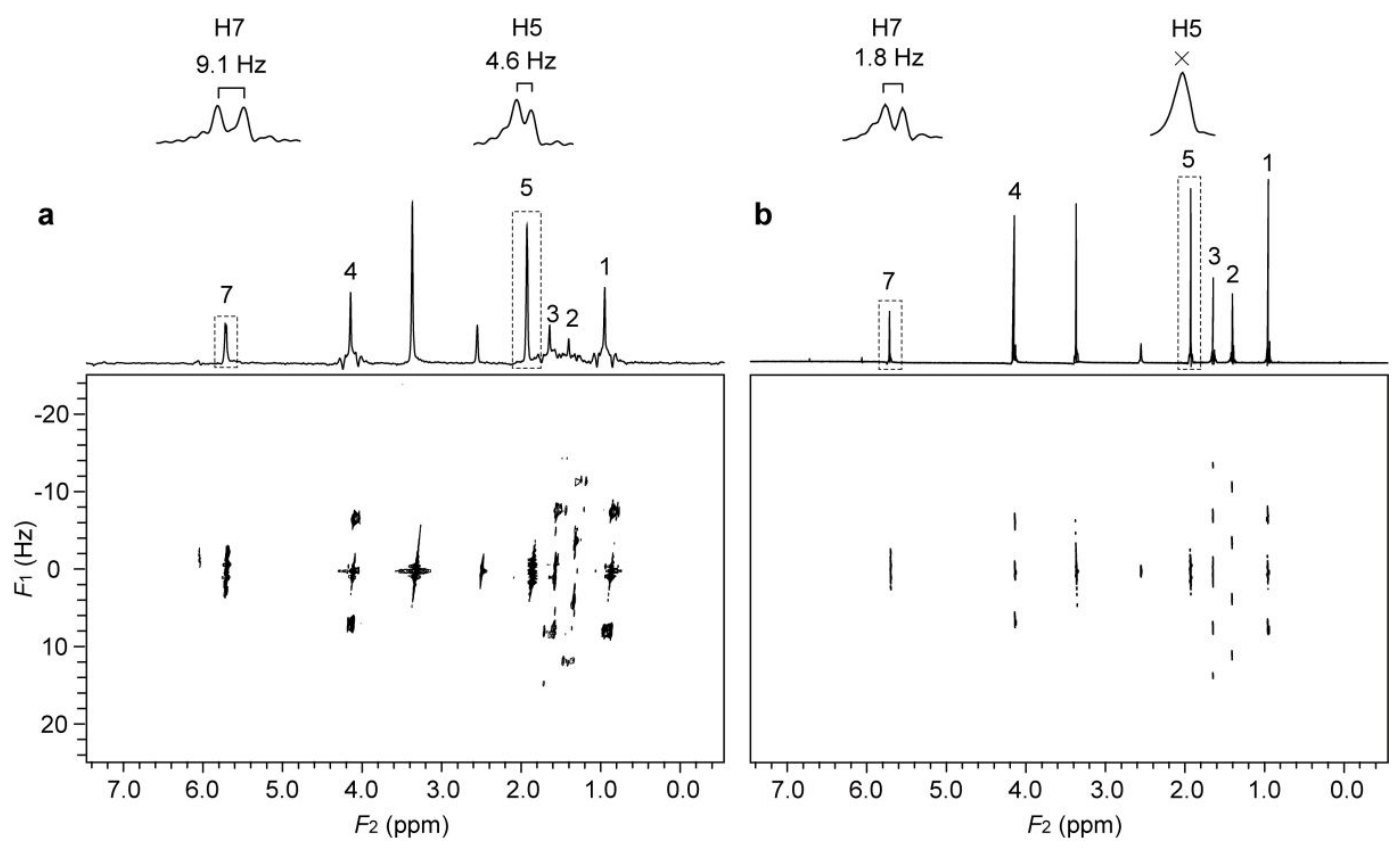

Figure S5. CASCADE spectra for butyl methacrylate with (a) and without (b) J-upscaling. The proton $\mathrm{H} 6$ was selected for investigation. $F_{2}$ projections are shown on top of $2 \mathrm{D}$ spectra. The J-upscaling factor is 5 . The small coupling constant between $\mathrm{H} 6$ and $\mathrm{H} 5$ cannot be resolved in b, while it can be extracted in a $(4.6 \mathrm{~Hz}$, corresponding to original value of $0.92 \mathrm{~Hz}$ ).

Selective Rsnob $180^{\circ}$ pulses were set to duration of $12.33 \mathrm{~ms}$ with theoretical bandwidth of about $150 \mathrm{~Hz}$, and were exerted on proton $\mathrm{H} 6$. Chirp pulses were employed with a sweep-width of $10 \mathrm{kHz}$ and a flip angle of $20^{\circ}$. The spectral width in $F_{1}$ dimension (SW1), the numbers of $t_{1}$ increments $\left(\mathrm{N}_{\mathrm{i}}\right)$, the amplitudes and duration of the spoiling gradients $\left(G_{1}, G_{2}, G_{3}\right.$ and $\left.t_{s}\right)$, the amplitude of gradient $G_{e}$, and the number of transients $\left(N_{t}\right)$ were set as SW1 $=50 \mathrm{~Hz}, \mathrm{~N}_{\mathrm{i}}=64, \mathrm{G}_{1}=23.19 \mathrm{G} / \mathrm{cm}, \mathrm{G}_{2}=14.65 \mathrm{G} / \mathrm{cm}, \mathrm{G}_{3}=9.16 \mathrm{G} / \mathrm{cm}, \mathrm{t}_{\mathrm{s}}=1$ $\mathrm{ms}, \mathrm{G}_{\mathrm{e}}=0.82 \mathrm{G} / \mathrm{cm}$, and $\mathrm{N}_{\mathrm{t}}=4$. The J-upscaling factor is 5 . The lengths of data chunks were approximately $10-20 \mathrm{~ms}(\mathrm{t} / \mathrm{n})$. The number of total acquisition points in the direct dimension was 3200 . The experimental duration of each experiment was about $160 \mathrm{~min}$.

Couplings along the $F_{1}$ dimension in the $J$-upscaled spectrum are not changed. The tilting angle of the $J$-upscaled spectrum is $\arctan (\lambda)$ ( $\lambda$ is the $J$-upscaling factor) rather than $45^{\circ}$ in 2D $J$ spectrum without $J$ upscaling. 


\section{Results of inosine for demonstration of suppression of strong coupling artifacts}

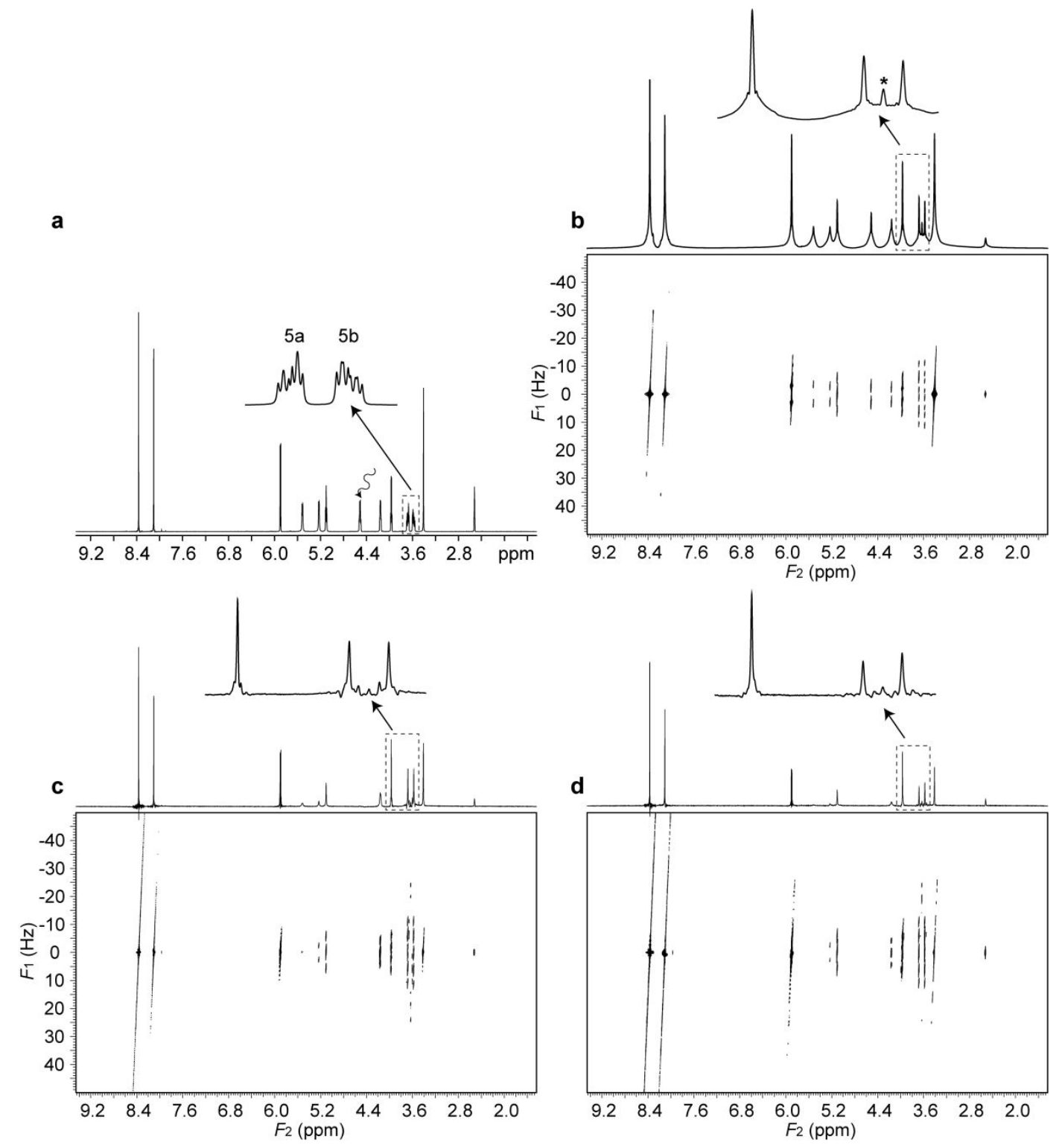

Figure S6. (a) Normal $1 \mathrm{D}{ }^{1} \mathrm{H}$ spectrum of inosine in DMSO- $d_{6}$, with close-up view of the region of protons $\mathrm{H} 5 \mathrm{a}$ and $\mathrm{H} 5 \mathrm{~b}$ with moderate strong coupling. (b) Conventional $2 \mathrm{D}$ J-resolved spectrum (magnitude-mode). (c) Regular CASCADE spectrum (absorption-mode). (d) CASCADE spectrum (absorption-mode) with better suppression of strong coupling artifacts by employing gradient-encoded chirp $180^{\circ}$ pulses. The $F_{2}$ projections are shown on top of 2D spectra. It can be seen that strong coupling artifact (marked by asterisk in b) in conventional 2D J-resolved experiment is reduced in CASCADE experiments. 


\section{Codes of pulse sequence}

\section{CASCADE-I:}

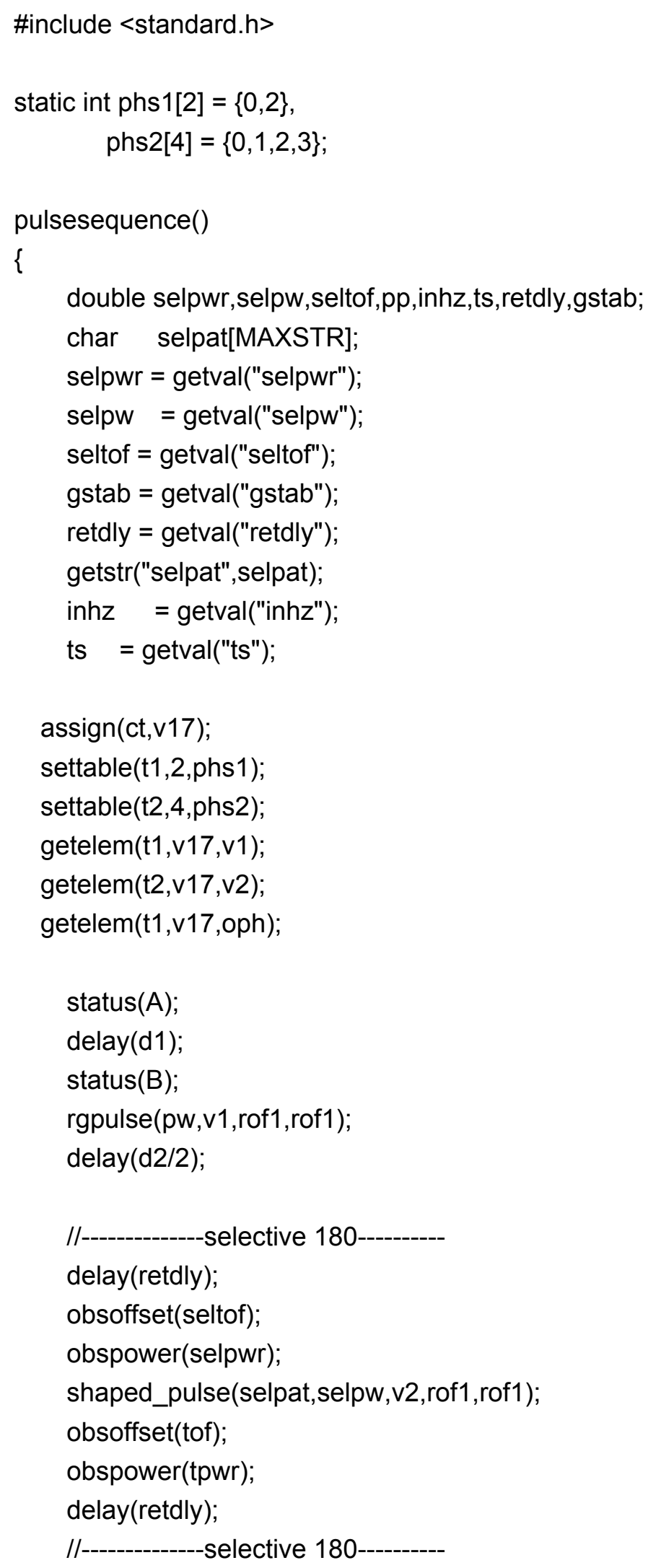




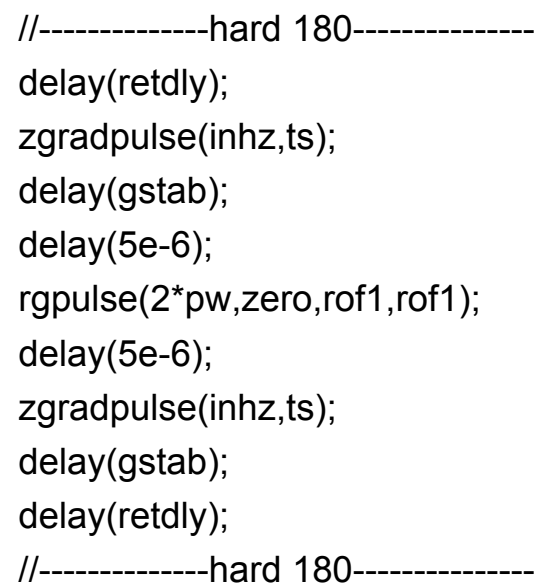

delay(d2/2);

//---------------selective 180----------

delay(retdly);

obsoffset(seltof);

obspower(selpwr);

shaped_pulse(selpat,selpw,zero,rof1,rof1);

obsoffset(tof);

obspower(tpwr);

delay(retdly);

//---------------selective 180----------

\section{CASCADE-II N-type:}

\#include <standard.h>

static int phs1[2] $=\{0,2\}$,

phs2[4] $=\{0,0,2,2\}$,

phs3[4] $=\{0,1,2,3\}$;

pulsesequence()

\{

double selpwr,selpw,seltof,pp,inhz,inhz1,inhz2,ts,ts1,ppwr,pdur,Ge,retdly,gstab;

char selpat[MAXSTR];

char ppat1[MAXSTR];

char ppat2[MAXSTR];

selpwr = getval("selpwr");

selpw = getval("selpw"); 


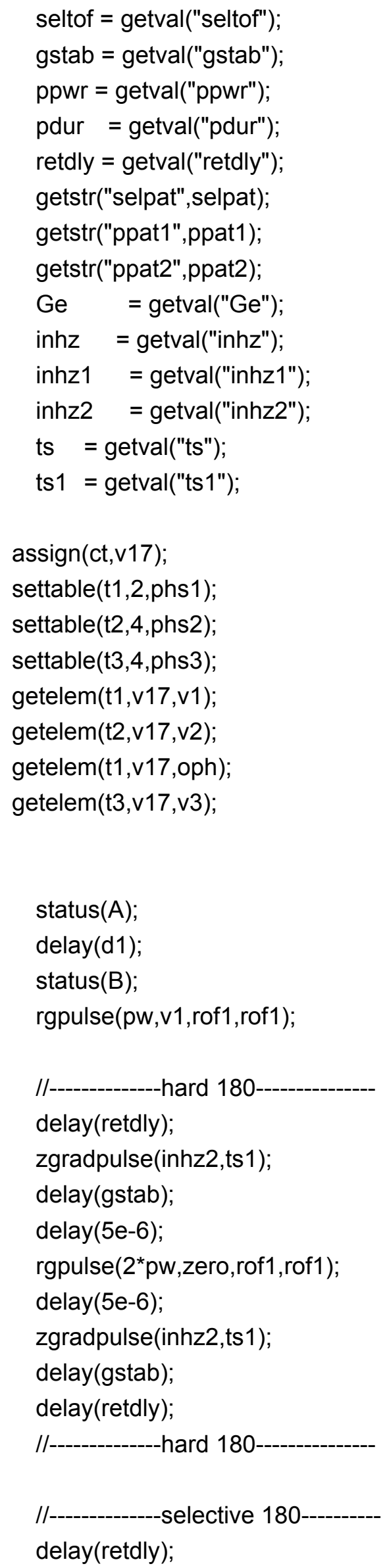




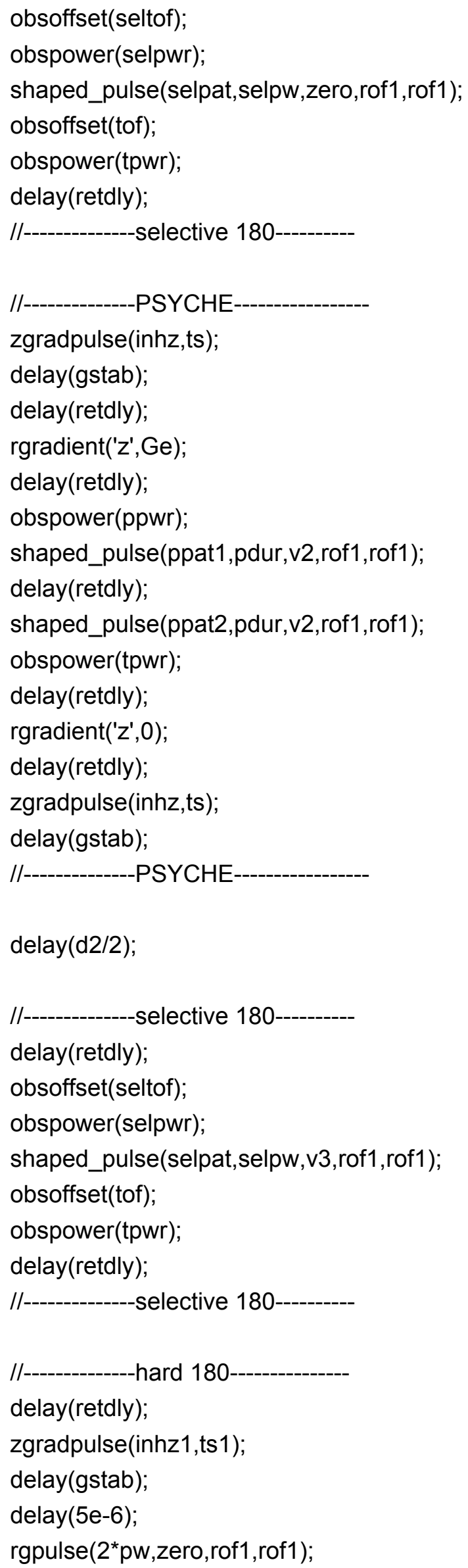


delay (5e-6);

zgradpulse(inhz1,ts1);

delay(gstab);

delay(retdly);

//-----------hard 180

delay(d2/2);

\section{CASCADE-II R-type:}

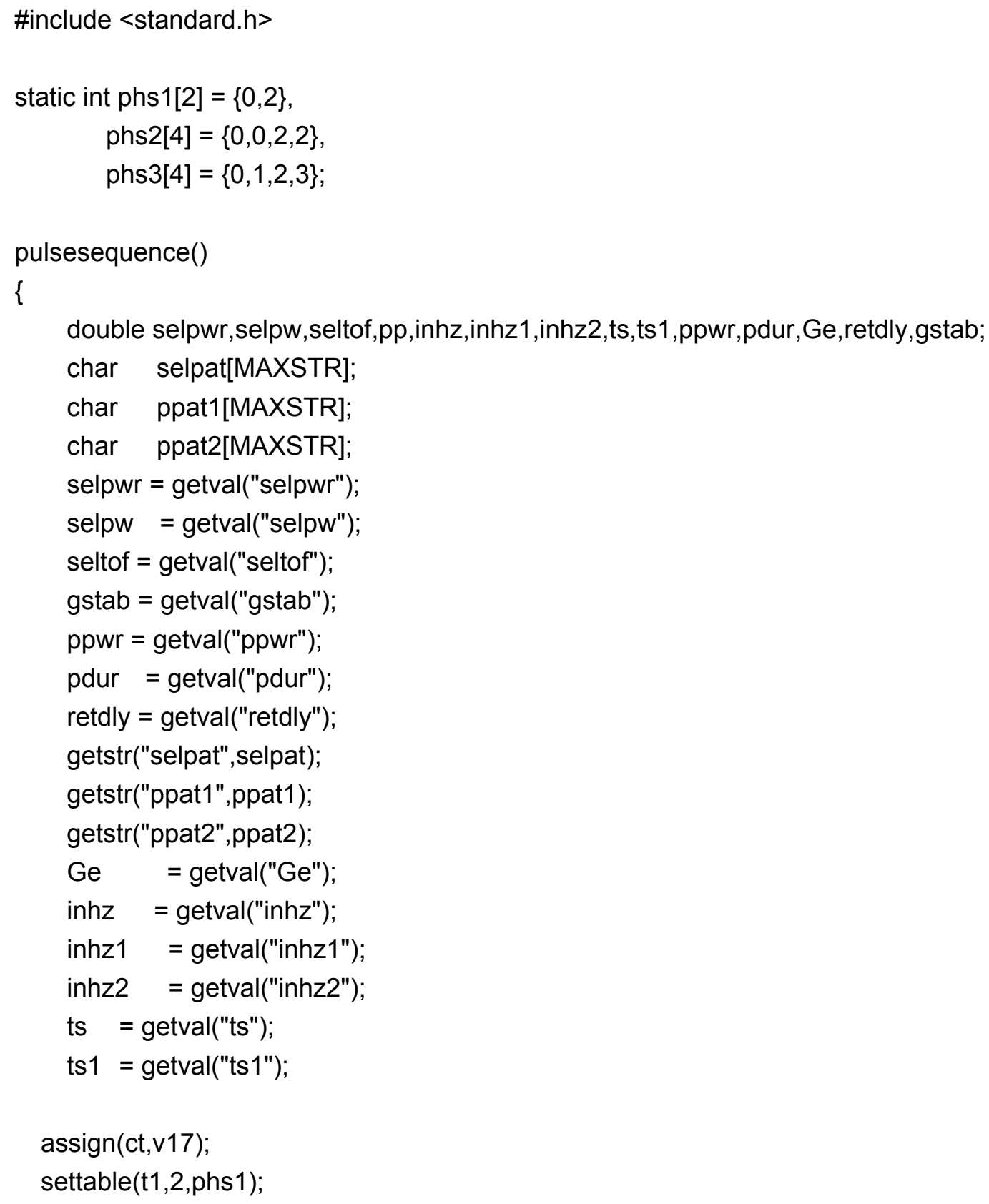


obspower(ppwr);

shaped_pulse(ppat1,pdur,v2,rof1,rof1);

delay(retdly);

shaped_pulse(ppat2,pdur,v2,rof1,rof1);

obspower(tpwr);

delay(retdly);

rgradient('z',0);

delay(retdly);

zgradpulse(inhz,ts);

delay(gstab);

//--PSYCHE

//-----------selective 180---------

delay(retdly);

obsoffset(seltof);

obspower(selpwr);

shaped_pulse(selpat,selpw,zero,rof1,rof1);

obsoffset(tof);

obspower(tpwr);

delay(retdly);

//---------------selective 180-----------

//-------------hard 180

delay(retdly);

zgradpulse(inhz2,ts1);

delay(gstab);

delay (5e-6);

rgpulse $\left(2^{*} \mathrm{pw}\right.$,zero,rof1, rof1);

delay(5e-6);

zgradpulse(inhz2,ts1);

delay(gstab);

delay(retdly);

//--------------hard 180 